Revisión de tema

Vol. 18(3):213-217, diciembre 2015 - marzo 2016

\title{
Revisión bibliográfica de la detección temprana de luxación incudomaleolar mediante imágenes tomográficas
}

\author{
Literature Review of Early Detection of Incudomalleolar \\ Dislocation by Using Tomographic Imaging \\ Revisão da literatura de detecção precoce do deslocamento \\ incudomallear por imagens tomográficas
}

\author{
Federico Lubinus-Badillo, MD., Esp. * \\ Evelyn Zuñiga-Hadechni, MD., Esp. ** \\ Lady Yadira Reyes-Álvarez, MD. *** \\ Ilich Fabián Plata-Cano, MD. **** \\ Jean Fabio Plata-Cano, MD. *****
}

\section{Resumen}

Introducción: Esta revisión hace énfasis en la luxación incudomaleolar. Aunque poco frecuente, es una patología con gran relevancia clínica debido a la afectación de la audición a corto y mediano plazo en los pacientes, afectando así su calidad de vida, por lo que es necesario un diagnóstico oportuno para un tratamiento temprano y eficaz. Objetivo: describir los hallazgos imagenológicos e identificar los diferentes mecanismos etiológicos de la luxación incudomaleolar. Metodología: Se realizó una revisión bibliográfica en PubMed de los artículos publicados hasta febrero de 2015 con las palabras claves: "Ear ossicles"; "Temporal bone"; "Bone"; "Fractures"; "Dislocation"; "Conductive hearing loss". Se encontraron 352 artículos de los cuales se seleccionaron aquellos donde se menciona la luxación incudomaleolar. Resultados: Se revisaron los abstracts de los 352 artículos, encontrando 20 donde mencionaban la definición, la epidemiología, la clínica y el diagnóstico imagenológico de la luxación incudomaleolar, los cuales fueron utilizados para la elaboración de esta revisión. Conclusiones: La principal causa de daño en la cadena de huesecillos es la fractura del hueso temporal y dentro de las patologías que puede generar está la luxación incudomaleolar, por lo que es de interés el diagnóstico temprano a todos los pacientes con sospecha clínica para llegar a prevenir complicaciones. La revisión de la literatura permite concluir que la técnica más eficaz para la identificación de la luxación Incudomaleolar es la tomografía computarizada, la cual requiere una adecuada identificación en los diferentes cortes multiplanares, o en las reconstrucciones 3D para poder diagnosticarla. [LubinusBadillo F, Zuñiga-Hadechni E, Reyes-Álvarez LY, Plata-Cano IF, Plata-Cano JF. Revisión bibliográfica de la detección temprana de luxación incudomaleolar mediante imágenes tomográficas. MedUNAB 2016; 18(3): 213-217]

Palabras clave: Osículos del oído; Hueso temporal; Fracturas óseas; Hueso; Pérdida auditiva conductiva.

\footnotetext{
* Federico Lubinus-Badillo, Médico y Cirujano, Especialista en Radiología e Imágenes Diagnósticas. Clínica Carlos Ardila Lülle, Colombia.

** Evelyn Zuñiga-Hadechni, Médico, Residente de Radiología. Clínica Carlos Ardila Lülle, Colombia.

*** Lady Yadira Reyes-Álvarez, Médico, Hospital Nuestra Señora de Guadalupe Santander, Colombia.

**** Ilich Fabián Plata-Cano, Médico, FOSCAL, Colombia.

*****Jean Fabio Plata-Cano, Estudiante de medicina VIII semestre, Universidad Autónoma de Bucaramanga, Colombia.
}

Autor de Correspondencia: Federico Lubinus-Badillo, Médico. Cll 155A23 09, Foscal torre A mod 15 Cañaveral, Floridablanca, Santander, Colombia. Correo electrónico: flubinus@unab.edu.co. 


\section{Abstract}

Introduction: This research emphasizes in the incudomallear dislocation. Although it is rare, it is a disease with great clinical relevance due to the short and long term involvement of patients' hearing so that it affects their quality of life, so it is very important a timely diagnosis for an early and effective treatment. Objective: To describe the imaging findings and identify the different etiologic mechanisms of incudomallear dislocation. Methodology: A literature review was carried out in PubMed about articles published until February 2015 with the keywords: "Ear ossicles"; "Temporal bone"; "Bone," "Fractures"; "Dislocation"; "Conductive hearing loss" finding 352 articles and selecting those that mentioned the incudomallear dislocation. Results: The abstracts of 352 articles were examined and 20 of them mentioned the definition, epidemiology, clinical and imaging diagnosis of incudomallear dislocation, and they were used to prepare this research. Conclusions: The main cause of damage to the ossicles is the temporal bone fracture, and among the diseases that this can produce, there is the incudomallear dislocation, therefore it is of great interest a timely diagnosis to all patients with clinical suspicion to prevent complications. The literature review allows us to conclude that the most effective technique to identify the incudomallear dislocation is computed tomography, which requires proper identification in different multiplanar cuts, or $3 \mathrm{D}$ reconstructions to diagnose it. [Lubinus-Badillo $F$, ZuñigaHadechni E, Reyes-Álvarez LY, Plata-Cano IF, Plata-Cano JF. Literature Review of Early Detection of Incudomalleolar Dislocation by Using Tomographic Imaging. MedUNAB 2016; 18(3): 213-217]

Key words: Ear ossicles; Temporal bone; Fractures, Bone; Conductive hearing loss.

\section{Introducción}

Respecto a la epidemiologia de la luxación incudomaleolar según datos obtenidos en el Servicio de radiología de la Clínica Alemana de Santiago de Chile, del Hospital San Juan de Dios de Costa Rica encontramos que aproximadamente un $30 \%$ de las fracturas del hueso temporal se asocian a compromiso de los huesecillos del oído (martillo, yunque y estribo) y de estas un 10 a $20 \%$ son de tipo extralaberínticas, comprometiendo el oído medio, externo o las estructuras situadas a este nivel (1). En los Estados Unidos las fracturas del peñasco del hueso temporal son más frecuentes en adultos jóvenes masculinos donde la causa más prevalente son los accidentes de tránsito, por lo que es de gran importancia sospechar de esta patología cuando se presenten traumatismos de alto impacto (2).

La articulación incudomaleolar es una articulación en la que se une la cara posterior de la cabeza del martillo con la cara anterior del cuerpo del yunque; es decir, que cuando ocurre alteración de dicha estructura y se genera la luxación incudomaleolar, ocurrirá alteración en la trasmisión de los estímulos vibratorios desde la membrana timpánica hasta el

\section{Resumo}

Introdução: Esta revisão enfatiza o deslocamento incudomallear. Embora seja raro, é uma doença de grande relevância clínica por causa do envolvimento da audição em pacientes a curto e a longo prazo, afetando sua qualidade de vida, por isso é muito importante o diagnóstico e assim poder realizar o tratamento precoce e eficaz. Objetivos: Descrever o diagnostico da imagem e identificar os diferentes mecanismos etiológicos da luxação incudomallear. Metodologia: A revisão da literatura foi realizada no PubMed para artigos publicados até fevereiro de 2015, com as palavras-chave: "ossículos do ouvido"; "Osso temporal"; "Osso", "fraturas"; "Deslocamento"; "A perda auditiva condutiva" foram encontrados 352 artigos e selecionados aqueles que mencionam o deslocamento incudomallear. Resultados: Foram revisados os resumos de 352 artigos e se encontrou que 20 artigos mencionavam a definição, epidemiologia, diagnóstico clínico e de imagem de incudomallear deslocamento, que foram utilizados para a preparação desta revisão. Conclusões: A principal causa de danos aos ossículos é a fratura do osso temporal e o deslocamento incudomallear é uma das patologias que poderia ocorrer, por esta causa é muito importante obter o diagnostico oportunamente e fazer o respectivo tratamento evitando então, graves complicações. A revisão da literatura permite-nos concluir que da técnica mais eficaz para a identificação de deslocamento incudomallear é a tomografia computadorizada, o que exige uma identificação adequada nos diferentes cortes multiplanares, ou reconstruções 3D para diagnosticar. [Lubinus-Badillo F, Zuñiga-Hadechni E, ReyesÁlvarez LY, Plata-Cano IF, Plata-Cano JF. Revisão da literatura de detecção precoce do deslocamento incudomallear por imagens tomográficas. MedUNAB 2016; 18(3): 213-217]

Palavras-chave: Ossículos do ouvido; Osso temporal; Fraturas ósseas; Osso; Perda auditiva condutiva.

estribo, generando finalmente disfunción de los huesecillos con posterior hipoacusia de conducción unilateral (3).

El oído es una estructura compleja localizada en el hueso temporal, en la fosa craneal media y debido a su localización intraósea las herramientas diagnósticas más utilizadas para su estudio son: la tomografía computarizada y la resonancia magnética, permitiendo así la visualización de cada una de sus estructuras anatómicas $(4,5)$. La tomografía computarizada es el examen de elección puesto que permite confirmar la presencia de fractura, observándose en ella una pérdida de las relaciones anatómicas normales de la articulación. Además, permite describir la orientación del trazo y precisar las distintas estructuras afectadas. Esta prueba de imagen se realiza por lo general en la fase aguda, principalmente en caso de síntomas clínicos como la otorragia, otorrea, hipoacusia de percepción o parálisis facial (6-7). El complejo incudomaleolar en las imágenes axiales se encuentra centrado con el receso epitimpánico del hueso temporal o lateral y la articulación incudomaleolar tiene la apariencia de un cono de helado donde la bola es la cabeza del martillo y el cono el proceso corto del yunque y el cuerpo (3). 
La luxación incudomaleolar, en la mayoría de los casos, se desencadena por traumas, específicamente los que afectan al hueso temporal y comprometen al oído medio, y de allí la cavidad que está llena de aire que es la que contiene los huesecillos, afectando particularmente al martillo y el yunque (1).

Las fracturas de la porción petrosa del hueso temporal o peñasco se dividen dependiendo de la posición de la fractura o la posición anatómica. Cabe recordar que tanto la primera como la segunda deben seguir el eje del peñasco hasta su extremo medial para que sea denominada fractura transversal, es decir, que sigue una línea perpendicular a dicho eje; de no cumplirse este criterio se denomina fractura oblicua, que es aquella que sigue un trayecto mixto Actualmente, sin embargo, la clasificación más utilizada es la clínica que divide las fracturas en laberínticas y extralaberínticas. Las laberínticas, también llamadas intralaberínticas, producen hipoacusia neurosensorial inmediata al traumatismo y se pueden asociar a vértigo; mientras que las extralaberínticas producen hipoacusia de transmisión, donde se encuentra afectada la parte mecánica del oído externo y medio, asociándose con frecuencia a otorragia (4-6). Los dos tipos de fracturas se pueden asociar a lesión del nervio facial si el trazo de la misma lo compromete en alguno de sus segmentos (6-10).

Las fracturas laberínticas generalmente son provocadas por traumas occipitales, son transversales y siguen la línea de fragilidad que atraviesa la fosita subarqueada, el vestíbulo y la ventana redonda. Las fracturas extralaberínticas suelen ser provocadas por traumatismos parietales que atraviesan la escama temporal y el conducto auditivo externo produciendo rotura timpánica y otorragia (8). Es importante aclarar que las lesiones de la cadena osicular o del laberinto membranoso se deben a un trauma del hueso temporal, pero no siempre deben ir acompañadas de fractura (11). En el oído medio la estructura más frecuentemente afectada es la cadena osicular, siendo de estas más frecuentes las luxaciones (93\%) que las fracturas (7\%) (4-5). La luxación incudoestapedial es la más frecuente en un $82 \%$ de los casos, y la luxación incudomaleolar en el $52 \%$, asociada en la mayoría de los pacientes a fracturas de la apófisis larga del yunque, del cuerpo del estribo o de presentarse concomitantemente con luxaciones incudoestapediales (1215).

\section{Metodología}

Se efectuó una revisión bibliográfica en PubMed de los artículos publicados hasta febrero de 2015 con las palabras claves: "Ear ossicles"; "Temporal bone"; "Bone"; "Fractures"; "Dislocation"; "Conductive hearing loss". Se encontraron 352 artículos de los cuales se seleccionaron aquellos donde fuera mencionada la luxación incudomaleolar. Se revisaron los abstracts de los 352 artículos encontrando 20 donde mencionaban la definición, la epidemiologia, la clínica y el diagnóstico imagenológico de la luxación incudomaleolar los cuales fueron utilizados para esta revisión.

\section{Discusión}

La prevalencia de la luxación incudomaleolar es baja debido a la solidez de la articulación, ya que el martillo posee inserciones sólidas caracterizándolo como el huesecillo más estable, mientras que el yunque es más pesado, pero menos estable porque tiene un débil soporte ligamentoso y no posee ningún tipo de inserción muscular propia, por lo que puede realizar rotaciones hasta de 180 grados alrededor del eje vertical y proyectarse hacia el tímpano, por esta razón, la luxación incudomaleolar puede provocar lesiones con secuelas otológicas de gran consideración (6). Por otro lado, la luxación incudoestapedial es mucho más frecuente ya que es una enartrosis frágil(11).

El trauma es considerado la etiología más frecuente de la luxación incudomaleolar, donde las fracturas del hueso temporal son las más habituales, especialmente la longitudinal extralaberíntica alcanzando el $80 \%$ de los casos frente al $4 \%$ de las fracturas transversas laberínticas, mientras que el $5 \%$ se relacionan con fracturas mixtas y en el $11 \%$ restante no se evidencia fractura del hueso temporal (3). Adicionalmente, se han descrito diversas modalidades en las que se puede presentar esta patología como en los casos de contusión cerrada, onda expansiva, heridas penetrantes o cuerpos extraños en el oído medio (1). Otra causa menos frecuente de luxación incudomaleolar son las malformaciones congénitas del oído medio; que tienen una incidencia de 1 por cada 10,000 habitantes, las cuales son unilaterales en un $70 \%$ de los casos, de predominio en el sexo masculino y de lateralidad derecha. Dentro de las malformaciones del oído medio, las que se asocian directamente con la luxación incudomaleolar son aquellas que afectan tanto a los huesos propios del oído, como a las estructuras de sostén de dicha articulación(16).

La luxación incudomaleolar se manifiesta clínicamente por una pérdida aguda unilateral de la audición de tipo conductiva, ya que las ondas sonoras no se pueden trasmitir en forma de energía mecánica al oído interno debido a la incapacidad de los huesecillos de vibrar al mismo tiempo (3). Este síntoma generalmente va acompañado de otorragia y hemotímpano debido al daño de diferentes estructuras internas del oído. Diferentes estudios han demostrado la relación entre el tipo de fractura de hueso temporal y el tipo de pérdida auditiva encontrando que las fracturas longitudinales están más asociadas al tipo conductivo mientras que las transversales al tipo neurosensorial, afectando la calidad de vida de quien las padece (14-20). En los casos donde se presenta compromiso de estructuras laberínticas se observa una asociación a vértigo e hipoacusia neurosensorial (3-5). El nervio facial puede estar comprometido con frecuencia hasta en un $80 \%$ de los casos, ocasionando complicaciones tempranas o tardías; cuando 


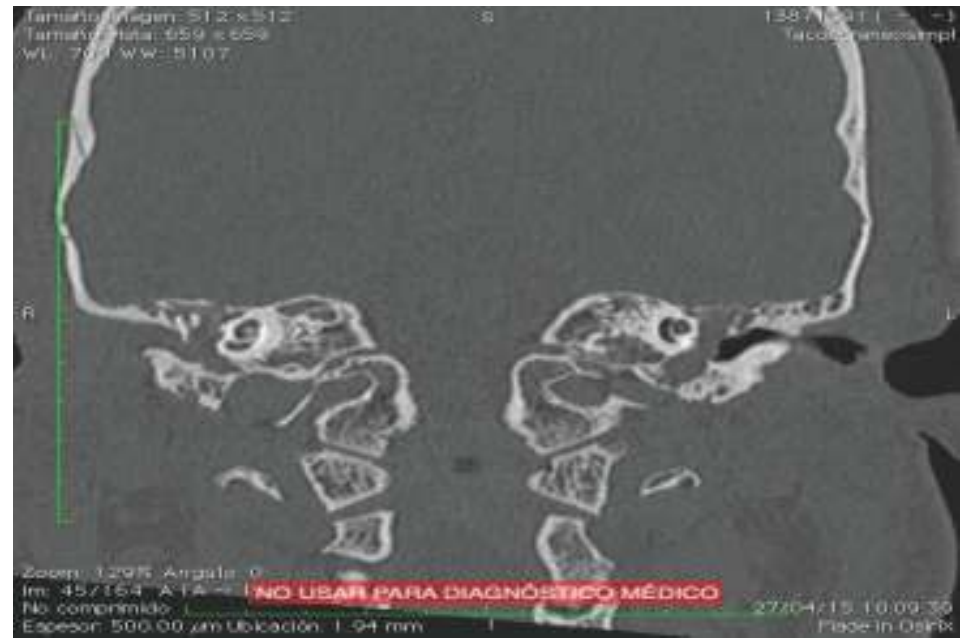

Fuente: Tomografía axial computarizada. Ilustración. Clínica Carlos Ardila Lulle, Floridablanca Santander.

Figura 1. Pérdida de relación entre cabeza de martillo con el proceso corto y cuerpo del yunque, Tomografía computarizada.

afecta su porción timpánica lleva a una parálisis facial de instauración inmediata al trauma ya que ha ocurrido una sección del nervio o compresión por un fragmento óseo, y se puede generar una parálisis diferida en el caso de edema o hematoma que comprima al nervio (9-10).

Por lo anteriormente dicho, se resalta la importancia de la detección temprana de estas lesiones por medio de la tomografía computarizada (4-5) (Figura 1). Además, se debe siempre sospechar de la presencia de la luxación incudomaleolar en aquellos casos donde se presente hipoacusia persistente a pesar de tener una membrana timpánica íntegra o tras la eliminación de detritus del conducto auditivo externo en caso de traumas con afectación de dichas estructuras (4-5).
Ante la sospecha de luxación incudomaleolar en primera instancia se debe realizar una exhaustiva historia clínica en donde el examinador observe algún hallazgo positivo al examen físico (otorragia, hemotímpano), se mencione algún tipo de antecedente o simplemente el paciente refiera algún síntoma relacionado en la revisión por sistemas como vértigo o hipoacusia. Posteriormente, se debe plantear la posibilidad de realizar una imagen diagnóstica que, en este caso, como primera opción, sería la tomografía computarizada con reconstrucciones multiplanares ya que permite un análisis adecuado de la cadena osicular. Existen diferentes técnicas como las reconstrucciones coronales oblicuas que son muy útiles para patología traumática y para visualizar la rama larga del yunque, las reconstrucciones axiales que son útiles para la visualización del estribo, y las reconstrucciones

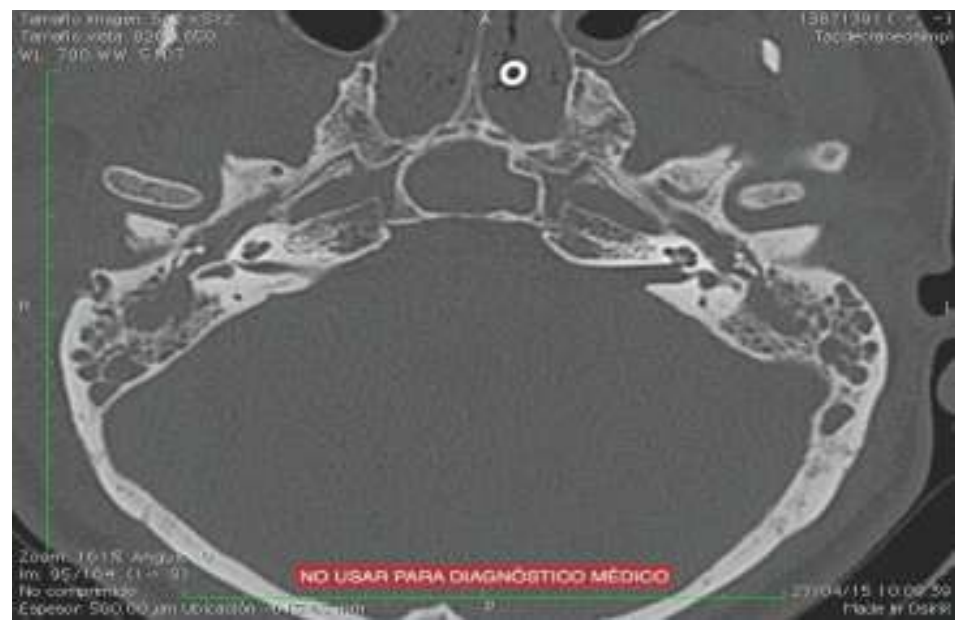

Fuente: Tomografía axial computarizada. Ilustración. Clínica Carlos Ardila Lulle, Floridablanca Santander.

Figura 2. Luxación incudomaleolar derecha y múltiples fracturas que se observa en la tomografía computarizada. 
sagitales que son las de mayor utilidad para identificar luxación incudomaleolar puesto que permiten detectar patologías del martillo y yunque (7). Estas diferentes técnicas se basan en detectar las discontinuidades anormales de dichas estructuras. En la tomografía computarizada se observa una imagen anatómica típica y característica que es "en cucurucho de helado", en donde hay una separación característica de la bola de helado (cabeza del martillo) respecto a su "cucurucho", que es la rama corta del yunque. La resonancia magnética solo está indicada en casos de sospecha de lesión cerebral, la cual debe constar al menos de una secuencia $\mathrm{T} 1$ con gadolinio centrada en las pirámides petrosas y una secuencia potenciada en $\mathrm{T} 2$ con cortes finos y contiguos (11).

Las fracturas laberínticas del hueso temporal nunca se consolidan, son de difícil manejo y son una vía de acceso para infecciones intracraneales graves (Figura 2). Las fracturas extralaberínticas, donde se encuentran frecuentemente las luxaciones incudomaleolares tienen un tratamiento quirúrgico funcional, de allí la importancia de un diagnóstico oportuno y precoz para la recuperación de la audición y prevención de complicaciones en el paciente como vértigo, hipoacusia neurosensorial o parálisis facial (8).

\section{Conclusiones}

La finalidad de esta revisión de tema es dar a conocer una patología poco frecuente, pero de gran importancia clínica ya que su detección temprana y certera tendrá como objetivo dar un tratamiento quirúrgico oportuno. Actualmente se considera a la tomografía computarizada como el método diagnóstico más eficaz para la identificación de la luxación incudomaleolar.

A pesar de que en nuestra población la prevalencia de accidentes de tránsito con compromiso de la tabla ósea es elevada, y el principal factor etiológico de esta patología sea el traumático, sigue siendo la luxación incudomaleolar una enfermedad poco frecuente debido a la solidez de esta articulación.

\section{Conflictos de intereses}

Los autores manifiestan que durante el desarrollo del trabajo no hubo conflictos de interés, tampoco hubo alguna fuente de financiación.

\section{Referencias}

1. González T, Mújica B, Larraín G, Miller P. Traumatismos del hueso temporal .Revista Chilena de Radiología. 2002; 8(4): 177-181.
2. Nevoux J, Nowak C, Benoudiba F, Bobin S. Fracturas del peñasco. EMC Otorrinolaringología. 2011;40(3): 1-21.

3. Meriot $P$, Veilon $F$, Mjean F, Nonent $M$, Jesequel J, Bourjat P, Belet M. CT appearances of ossicular injuries.Radiographics. 1997; 17(1): 445-54.

4. Offiah CE, Ramsden RT, Gillespie JE. Imaging appearances of unusual conditions of the middle and inner ear. Br J Radiol. 2008;81(966): 504-14.

5. Zayas JO, Feliciano YZ, Hadley CR, Gómez AA, Vidal JA. Temporal bone trauma and the role of multidetector CT in the emergency department.Radiographics. 2011;(6):1741-55.

6. Trujillo M, Palacios E, Valvassori G. Taveras JM. Imagenologia del hueso temporal y ángulo pontocerebeloso. Radiología e imagen diagnóstica y terapéutica, tomo 1.Philadelphia: Lippincott- Williams and Wilkins, 1999.

7. Cyna-Gorse F, Rodallec M, Bouccara D. Pruebas de imagen del oído medio normal y patológico. EMC Otorrinolaringología. ElsevierMasson SAS. 2009; 38(3): 1-21.

8. Trujillo M. Imagen diagnóstica en otoneurología. ARS médica. 2005

9. Asha'Ari, Z.A., Ahmad, R., Rahman, J. et al. Contrecoup injury in patients with traumatic temporal bone fracture. $\mathrm{J}$ Laryngol Otol. 2011; 125: 781-785.

10. (10). Carlsson PI, Hall M, Lind KJ, Danermark B. Quality of life, psychosocial consequences, and audiological rehabilitation after sudden sensorineural hearing loss. Int JAudiol. 2011; 50(2): 139-144.

11. Poirrier V, Escude B, Granier I, Langlois C, Bonafe A.Exploración radiológica de los traumatismos del hueso temporal. EMC otorrinolaringología. Elsevier. 2010.

12. Homassin J, Dessi P, Danvin J, Forman C. Anatomía del oído medio. EMC Otorrinolaringología. 2008;37(3): 1-20

13. Chavelier E, Courtat P, Parvy F, Peytral. Secuelas otológicas de los traumatismos craneocervicales. EMC Otorrinolaringología. 2006; 35(1): 1-19.

14. So Young K, Yoon Joong K, Young Ho K, Min-Hyun Park. Audiologic Patterns of Otic Capsule Preserving Temporal Bone Fracture: Effects of the Affected Subsites. Clin Exp Otorhinolaryngol. 2016 Sep; 9(3): 206-211. DOI:10.21053/ceo.2015.01116

15. Blake DM, Tomovic S, Jyung RW. Temporal bone fracture. Ear Nose Throat J. 2014; 93(1):16-25.

16. Teissier N, Benchaa T, Elmaleh M, Van Den Abbeele T.Malformaciones congénitas del oído externo y del oído medio. EMC Otorrinolaringología. 2008;37(4):1-11

17. Sun GH, Shoman NM, Samy RN, Cornelius RS, Koch $\mathrm{BL}$, Pensak ML. Do contemporary temporal bone fracture classification systems reflect concurrent intracranial and cervical spine injuries?. Laryngoscope. 2011; 121(5):929-32.

18. (18). Montava M, Mancini J, Masson C, Collin M, Chaumoitre K, Lavieille JP. Temporal bone fractures: sequelae and their impact on quality of life. Am J Otolaryngol. 2015;36(3):364-70.

19. Asha'ari Z.A, Ahmad R, Rahman J. et al. Patterns of intracranial hemorrhage in petrous temporal bone fracture. Auris Nasus Larynx. 2012; 39:151-155.

20. Stachler, R.J, Chandrasekhar, SS, Archer, SM. et al. Clinical practice guideline: sudden hearing loss. Otolaryngol Head Neck Surg. 2012; 146: s1-s35. 